\title{
Correction to: Formal Methods and Software Engineering
}

\author{
Shang-Wei Lin (D), Zhe Hou (ID, and Brendan Mahony
}

\section{Correction to: \\ S.-W. Lin et al. (Eds.): Formal Methods and Software \\ Engineering, LNCS 12531, \\ https://doi.org/10.1007/978-3-030-63406-3}

In the original version the surname of the editor Brendan Mahony has been misspelled. This has been now corrected. 